\section{Slicing and Dicing Pancreatic Cancer Into Actionable Nuggets}

\author{
Anirban Maitra, MBBS
}

Pancreatic ductal adenocarcinoma (PDAC) has a dismal 5-year survival rate of $9 \%{ }^{1}$ During the past few years, several large publicly funded initiatives, such as The Cancer Genome Atlas and the International Cancer Genome Consortium, ${ }^{2}$ as well as smaller single institution studies, ${ }^{3,4}$ have elucidated the comprehensive genomic landscapes of PDAC. These studies have shown that oncogenic KRAS mutations remain the dominant alteration in PDAC, with loss of function mutations of TP53, SMAD4, and CDKN2A rounding off the "big 4" mutations in this disease. Despite the preponderance of these 4 mutational events (each occurs in $>50 \%$ of tumors), unfortunately none are currently actionable, although considerable preclinical efforts are ongoing for developing antagonists to oncogenic KRAS or the encoded mutant protein. This does not, however, preclude the possibility of other lower-frequency alterations in the PDAC genome that remain eminently actionable. In fact, it has been estimated that as many as $40 \%$ of PDAC cases might harbor as many as one actionable alteration based on the current slate of therapeutic agents targeting specific mutations. ${ }^{5}$ In the accompanying article by Singhi et al (elsewhere in this issue), the authors queried targeted next-generation sequencing (NGS) offered through a commercial fee-forservice provider (Foundation Medicine, Cambridge, MA) in 3,170 PDAC cases and identified 5 PDACs (representing $0.16 \%$ of cases) with a somatic alteration in the anaplastic lymphoma kinase (ALK) gene. Remarkably, all 5 cases occurred in patients $<50$ years of age (by comparison, the median age of onset for PDAC is 65 years), and when specifically stratified by age of onset $<50$ years, the frequency of ALK gene alterations increased to $1.3 \%$, suggesting preferential occurrence in younger-onset disease. Further parsing of the genomic alterations demonstrated absence of KRAS mutations in all 5 cancers, which is striking, given the near ubiquitous nature of this abnormality in PDAC. Although this raises the specter of a pancreatic metastasis from an ALK-mutant lung primary, radiologic and clinical parameters were most consistent with a PDAC; expectedly, consistent with the generally advanced presentation of PDAC, resected tissue was available in only 1 of 5 cases. Notably, 4 of the 5 patients received one or more small molecule ALK inhibitors approved for ALK-mutant lung cancer (crizotinib, ceritinib, or alectinib), ${ }^{6}$ and 3 of them demonstrated at least some degree of objective response, based on imaging or biochemical parameters.

There are several pertinent messages one can distill from this study. First, actionable mutations that lead to objective responses can occur in an exceedingly small fraction of cases, such as the $0.16 \%$ of somatic ALK mutations observed here. However, it might be possible to enrich for this "zebra" by using simple clinical parameters, such as young age at diagnosis, which pushes the rate of positivity to $1.3 \%$, a prevalence that matches what is observed in lung cancer overall, and justifies the cost-effectiveness of targeted NGS panels for mutation profiling.

Second, this approach has a clinical rationale given the ready availability of both first- and second-generation ALK inhibitors, which can be administered once patients have experienced disease progression on current, standard-of-care, first-line cytotoxic chemotherapy regimens. At the same time, one must be cognizant of the fact that, in the current study, we are presented with incomplete data on outcomes, and the rate of sustained response to ALK inhibitors in ALK-mutant PDAC remains unknown.

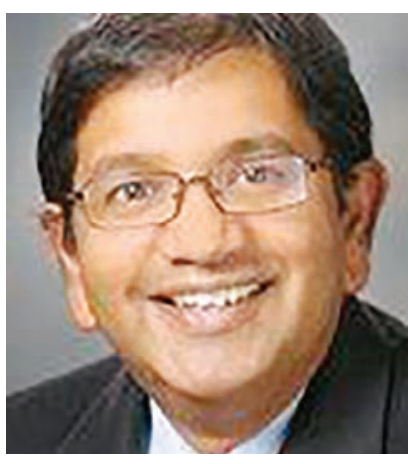

Anirban Maitra, MBBS

Dr. Anirban Maitra is Professor of Pathology and Translational Molecular Pathology, Sheikh Khalifa Bin Zayed Al Nahyan Distinguished University Chair, and Scientific Director of the Sheikh Ahmed Center for Pancreatic Cancer Research at the University of Texas MD Anderson Cancer Center in Houston, Texas. 
In order to garner a more complete story, we need to evaluate this agent in the context of a clinical trial. Yet, what would entice a pharmaceutical company to run a clinical trial in a disease in which the actionable subset is $0.16 \%$ ? This is where so-called "basket trials" become particularly pertinent, and not just because they allow for a reasonably rapid accrual of a respectable number of cancers harboring a defined genetic alteration. ${ }^{7}$ A basket trial also allows for separation of organ- and context-specific efficacy when distinct tumor types are treated with an identical agent, as recently shown in a trial of nonmelanoma BRAF-mutant cancers treated with vemurafenib. ${ }^{8}$ Within the PDAC genomic landscape, probably the most prevalent actionable subtype (currently estimated at between $5 \%$ and $10 \%$ of cases) is composed of tumors with defective DNA damage repair ("DDR-defective" PDAC), which respond to platinum agents and poly(ADP-ribose) polymerase inhibitors. ${ }^{9}$ Beyond DDR-defective PDAC, many other low frequency alterations $(<5 \%$, and typically $<2 \%$ ) now exist with available small molecule inhibitors or biologics, including mutations of BRAF and EGFR or amplifications of HER2/neu, and with the plummeting cost of NGS and availability of basket trials around the country, hunting for such actionable subsets, minor as they might be, becomes increasingly justifiable.

Third, from a biological perspective, the absence of near ubiquitous KRAS mutations in all $5 \mathrm{ALK}$-positive cases suggests that the pathogenesis of these lesions may be distinct from garden variety PDAC. For example, it was not clarified whether the pancreata contain bona fide precursor lesions, such as pancreatic intraepithelial neoplasia, which is not surprising given that resectable material was available from only 1 case. Mutant Ras signaling leads to profound immunosuppression in the tumor microenvironment, ${ }^{10}$ and in its absence, the theoretical possibility remains that KRAS wild-type cases might be more susceptible to immunotherapy.

Finally, as a cautionary note, in clinical oncology, common things happen commonly, and in patients who present with a pancreatic "mass" that is KRAS wildtype and ALK-mutant, the first possibility that needs to be excluded is that of a pancreatic metastasis from a lung primary. As noted earlier, the authors acknowledge this limitation, although clinical and imaging data strongly support a pancreatic primary. A robust line of communication between the oncology, radiology, and pathology teams is critical for ensuring an accurate diagnosis in such cases.

\section{References}

1. Rahib L, Smith BD, Aizenberg R, et al. Projecting cancer incidence and deaths to 2030: the unexpected burden of thyroid, liver, and pancreas cancers in the United States. Cancer Res 2014;74:2913-2921.

2. Bailey $P$, Chang $D K$, Nones $K$, et al. Genomic analyses identify molecular subtypes of pancreatic cancer. Nature 2016;531:47-52.

3. Jones S, Zhang X, Parsons DW, et al. Core signaling pathways in human pancreatic cancers revealed by global genomic analyses. Science 2008;321:1801-1806.

4. Witkiewicz AK, McMillan EA, Balaji U, et al. Whole-exome sequencing of pancreatic cancer defines genetic diversity and therapeutic targets. Nat Commun 2015;6:6744.

5. Dreyer SB, Chang DK, Bailey P, Biankin AV. Pancreatic cancer genomes: implications for clinical management and therapeutic development. Clin Cancer Res 2017;23:1638-1646.

6. Holla VR, Elamin YY, Bailey AM, et al. ALK: a tyrosine kinase target for cancer therapy. Cold Spring Harb Mol Case Stud 2017;3:a001115.

7. Hyman DM, Taylor BS, Baselga J. Implementing genome-driven oncology. Cell 2017;168:584-599.

8. Hyman DM, Puzanov I, Subbiah V, et al. Vemurafenib in multiple nonmelanoma cancers with BRAF V600 mutations. N Engl J Med 2015;373:726-736.

9. Vyas $\mathrm{O}$, Leung $\mathrm{K}$, Ledbetter $\mathrm{L}$, et al. Clinical outcomes in pancreatic adenocarcinoma associated with BRCA-2 mutation. Anticancer Drugs 2015;26:224-226.

10. Vonderheide RH. Tumor-promoting inflammatory networks in pancreatic neoplasia: another reason to loathe Kras. Cancer Cell 2014;25:553-554. 